\title{
An Investigation into the Effective Heat Transfer Coefficient in the Casting of Aluminum in a Green-Sand Mold
}

\author{
Hsien-Chi Sun and Long-Sun Chao* \\ Department of Engineering Science, National Cheng Kung University, No. 1, University Rd., Tainan City 701, Taiwan, R.O.China
}

\begin{abstract}
This study commences by performing an experimental investigation to measure the temperature distribution within a casting system comprising a cylindrical aluminum casting and a green-sand mold. The experimental temperature measurements are then used to compute the effective heat transfer coefficient at the mold/metal interface using four different formulae. In the temperature measurement, a symmetric arrangement of thermocouples is proposed and proven to be feasible, which can reduce the influence of heat-transfer and solidification characteristics in a casting experiment due to the close-spaced thermocouples. As an important role in the calculation of the effective heat transfer coefficient, the metal temperature at the mold/metal interface is calculated using an extrapolation technique and an inverse scheme. A lump capacity method is also utilized to estimate the average values of the effective heat transfer coefficients, which are consistent with those of the previous effective heat transfer coefficients. The numerical results obtained for the temperature curves in the green-sand mold are found to agree well with the experimental profiles. Finally, with the effective heat transfer coefficients obtained above, a finite element simulation is performed using FIDAP software to model the evolution of the temperature distribution within the casting during the solidification process. The predicted solidification time is found to be in reasonable agreement with that observed in the experimental casting process.

[doi:10.2320/matertrans.MRA2008364]
\end{abstract}

(Received October 14, 2008; Accepted March 2, 2009; Published May 25, 2009)

Keywords: heat transfer analysis, sand mold casting, gap, mold and melt interface

\section{Introduction}

The temperature variations induced in a cast product have a direct influence on its final microstructure, and therefore play a critical role in determining its quality. Consequently, analyzing the heat transfer characteristics of the casting system is of crucial importance. In analyzing the solidification of cast products, the accuracy of the results is governed to a large degree by the manner in which the heat transfer conditions at the mold/metal interface are modeled. The simplest approach is to assume that the molten metal and the mold form a perfect contact such that the temperature and the heat flux distributions are continuous at the interface. However, in practice, this assumption is invalid since a narrow air gap inevitably exists between the metal and the mold as a result of thermal expansion, the vaporization of additives in the sand mold, the discharge of air from the mold cavity during the pouring process, and so on. This air gap acts as an insulating layer between the molten metal and the mold and thus an effective heat transfer coefficient is required to compute the true value of the heat flux transferred through the mold/metal interface. Thus, the literature contains many experimental and numerical investigations aimed at analyzing the heat transfer characteristics at the mold/metal interface of typical casting systems and deriving appropriate formulations for the corresponding effective heat transfer coefficient.

Ho and Pehlke ${ }^{1)}$ investigated the interfacial heat transfer characteristics of two similar casting systems. In the first instance, a copper chill was placed on top of a cylindrical bottom-gated casting, while in the second, the chill was placed beneath the casting. In the first case, a small clearance gap was detected between the casting and the chill following the solidification process. The influence of the interfacial heat

*Corresponding author, E-mail: 1schao@mail.ncku.edu.tw transfer on the solidification time was examined numerically for three different mold materials and the results were then used to establish suitable criteria for Chvorinov's solidification rule. Zeng and Pehlke ${ }^{2)}$ and Hou and Pehlke ${ }^{3)}$ analyzed the interfacial heat transfer characteristics of cylindrical gray iron and aluminum-silicon castings, respectively, and showed that the numerical results obtained for the temperature distribution and solidification time were in good agreement with the experimental observations.

Kubo and Pehlke ${ }^{4)}$ performed an experimental investigation into the transfer of heat and moisture in sand molds and used the empirical data to construct a model to compute the rate of movement of the dry/wet mold interface. Tsai et al. ${ }^{5)}$ Shih et al. ${ }^{6)}$ and Marek ${ }^{7)}$ used numerical methods to calculate the temperature distribution within sand-mold casting systems and to predict the motion of the dry/wet interface as a function of the original moisture content of the sand mold. Kobryn and Semiatin ${ }^{8)}$ measured the cooling curve of a Ti$6 \mathrm{Al}-4 \mathrm{~V}$ casting produced was used a finite element method based upon an estimated value of the thermal resistance at the mold/metal interface to reproduce the experimental thermal distribution. In an iterative process, the estimated value of the thermal resistance was progressively refined by comparing the computed cooling curve with the experimental curve. The iterations continued until the difference between the two curves satisfied a pre-determined termination criterion, at which point, the current value of the estimated thermal resistance was taken to represent the true value of the effective heat transfer coefficient at the mold/metal interface. Nishida and Semiatin ${ }^{9)}$ used the explicit finite different method and an estimated value of the heat transfer coefficient to compute the temperature distributions in cylindrical and flat aluminum castings. The heat transfer coefficient was corrected iteratively by comparing the computed temperature value with the experimental value until a convergent coefficient was obtained. The authors utilized an extrapola- 
tion technique to calculate the metal and mold temperatures at the mold/metal interface based upon the measured temperature values near the interface. The extrapolated interface temperature was then used to derive the corresponding values of the heat flux and heat transfer coefficient, respectively. Hwang et al. ${ }^{10)}$ conducted a similar investigation using an A356 aluminum alloy casting in a sand mold. Utilizing an inverse technique, Browne and O'Mahoney ${ }^{11)}$ showed that the interfacial thermal resistance in an investment casting process was related to the solidification range of the alloy, the hydrostatic pressure of the liquid metal, the thickness of the investment shell, the pre-heat conditions, and the interface geometry. Chiesa ${ }^{12)}$ measured the temperature of the molten metal during the filling of a flat plate mold, and concluded that the difference between the measured temperature value and the numerically-derived temperature value implied the presence of a thermal resistance at the mold/ metal interface even though the metal was in a molten state.

From the discussion above, heat resistance does exist at the sand mold/metal interface. Although the studies presented above confirm the existence of a thermal resistance at the mold/metal interface in casting systems, they do not present simple generic formulae for deriving the value of the effective heat transfer coefficient at particular mold/metal interfaces. Consequently, this study conducts an experimental casting process in which a cylindrical aluminum product is cast in a green-sand mold, and then uses the experimental temperature measurements to derive the value of the effective heat transfer coefficient at the mold/metal interface using four different analytical formulae. In the temperature measurement, a symmetric arrangement of thermocouples is proposed and proven to be feasible, which can reduce the influence of heat-transfer and solidification characteristics in a casting experiment caused by the close-spaced thermocouples. As a key role in the calculation of the effective heat transfer coefficient, the metal temperature at the mold/metal interface is calculated using an extrapolation technique and an inverse method. The experimental cooling curves in the green-sand mold are compared with the numerically-derived profiles. The heat transfer phenomena within the casting are modeled as a lump capacity problem, which is then solved to estimate the average value of the effective heat transfer coefficient. The average values of the lump method are also compared with those of the previous effective heat transfer coefficient. Finally, the effective heat transfer coefficient is substituted into a commercial finite element software program (FIDAP) and used to compute the temperature field within the casting during the solidification process. The validity of the simulation model is confirmed by comparing the predicted value of the solidification time with that measured experimentally.

\section{Experimental and Modeling Methods}

The objective of this study is to investigate the effective heat transfer coefficient at the mold/metal interface during the casting of a cylindrical aluminum product in a green sand mold. The effective heat transfer coefficient is calculated in accordance with the temperature data measured during the solidification process. The temperature of the metal at the mold/metal interface is derived using inverse and extrapolation methods due to the physical difficulty of embedding a thermocouple at the interface position. A numerical method is then employed to compute the temperature distribution within the green sand mold during the casting process. The validity of the numerical results is confirmed by comparing the predicted value of the solidification time with the experimentally observed result.

\subsection{Casting experiment with symmetric arrangement of thermocouples}

The casting experiment performed in this study considered the case of a cylindrical casting with a diameter of $4.4 \mathrm{~cm}$ and a length of $14.7 \mathrm{~cm}$ in a green-sand mold. The casting material was $99.86 \%$ pure aluminum. In casting experiments, the number and positions of the thermocouples used to measure the temperature distributions within the casting and the mold, respectively, must be carefully controlled to avoid affecting the heat-transfer, filling and solidification characteristics of the casting system. For example, a dense arrangement of low-temperature thermocouples prompts a local artificial solidification effect which has a significant effect on the characteristics of the remainder of the casting process. However, the measurement positions in the casting and the mold should ideally be spaced as closely as possible in order to ensure the continuity of the measured temperature profiles, thereby improving the accuracy of both the estimated interfacial temperature and the computed value of the effective heat transfer coefficient. To satisfy these conflicting requirements, the current study measured the temperature distributions within the casting and the greensand mold proposing a symmetrical arrangement of thermocouples, in which one thermocouple placed at a designated measurement position was "balanced" by a second thermocouple installed in a symmetrical location with respect to the centerline of the casting system. As indicated in Fig. 1(a), thermocouples were installed along the pattern line of the metal and in the sand mold whose locations were symmetrical to the centerline. Figure 1(b) and 1(c) illustrate the measured temperatures at symmetrical points in casting and mold. In these figures, the temperatures recorded at measurement points positioned in symmetrical locations in the casting and the green-sand mold were found to be virtually identical, and thus the suitability of the thermocouple configuration is confirmed. Figure 2 presents a schematic illustration of the four measurement points near the mold/ metal interface used to calculate the effective heat transfer coefficient. As shown, $\mathrm{x}_{1}$ and $\mathrm{x}_{2}$ are located in the casting, while $\mathrm{x}_{3}$ and $\mathrm{x}_{4}$ are located in the green-sand mold. Note that in this figure, the notations $\mathrm{x}_{\mathrm{i}}$ and $T_{\mathrm{i}}$ denote the $\mathrm{x}$-coordinates and temperatures of the measurement points, respectively, while $T_{\text {metal }}$ and $T_{\text {sand }}$ indicate the temperatures of the metal and the green-sand mold at the interface, respectively.

Figure 3 illustrates the positions of the various thermocouples used in the casting experiment to measure the temperature distributions in the interfacial region of the casting system and in the interior of the casting, respectively. Note that in this figure, points $\mathrm{a}, \mathrm{b}, \mathrm{c}$ and $\mathrm{d}$ correspond to points $\mathrm{x}_{1}, \mathrm{x}_{2}, \mathrm{x}_{3}$ and $\mathrm{x}_{4}$ in Fig. 2. In the casting experiment, it is impossible to measure the metal temperature at the mold/ 


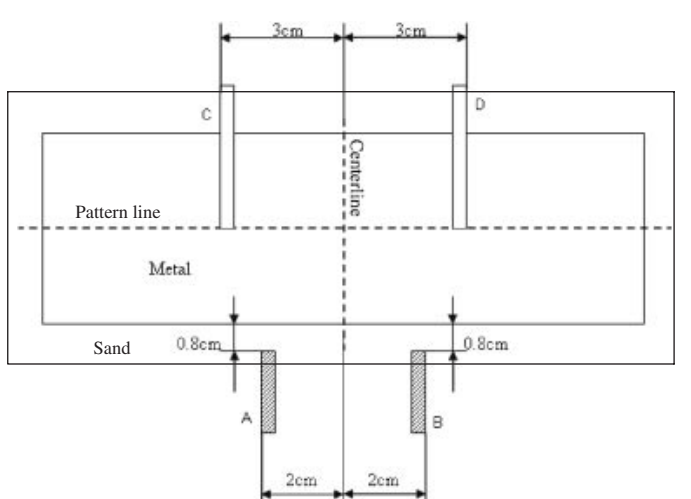

(a) Thermocouples installed along the pattern line of the metal and in the sand mold whose locations are symmetrical to the centerline.

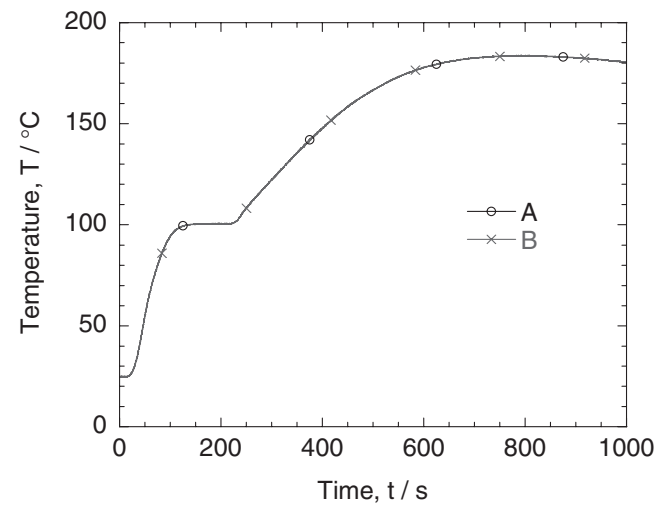

(b) Measured temperatures at symmetrical points A and B in green-sand mold.

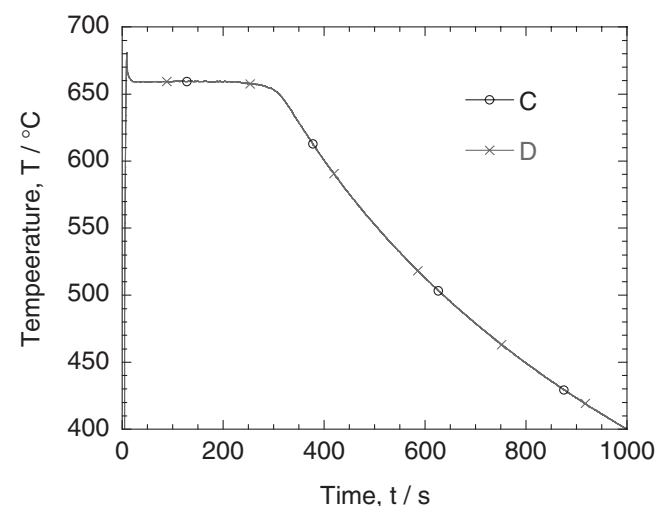

(c) Measured temperatures at symmetrical points $\mathrm{C}$ and $\mathrm{D}$ in metal.

Fig. 1 Experimental temperature profiles at centerline-symmetrical measured positions in metal and green-sand mold, (a) Thermocouples installed along the pattern line of the metal and in the sand mold whose locations are symmetrical to the centerline, (b) Measured temperatures at symmetrical points $\mathrm{A}$ and $\mathrm{B}$ in green-sand mold, (c) Measured temperatures at symmetrical points $\mathrm{C}$ and $\mathrm{D}$ in metal.

metal interface $\left(T_{\text {metal }}\right)$ directly using a thermocouple. Consequently, the temperature data measured at points $\mathrm{x}_{1}$ and $\mathrm{x}_{2}$, i.e. $T_{1}$ and $T_{2}$, are used to calculate $T_{\text {metal }}$ using either an extrapolation technique or a numerical inverse method, respectively. Having calculated this temperature, the heat flux exiting the metal casting at the interface, i.e. $q_{\text {metal }}$, can then be estimated. Similarly, $q_{\text {sand }}$ is determined from temperature measurements $T_{3}\left(T_{\text {sand }}\right)$ and $T_{4}$. Note that in contrast to $T_{\text {metal }}, T_{\text {sand }}$ (corresponding to position $\mathrm{x}_{3}$ in

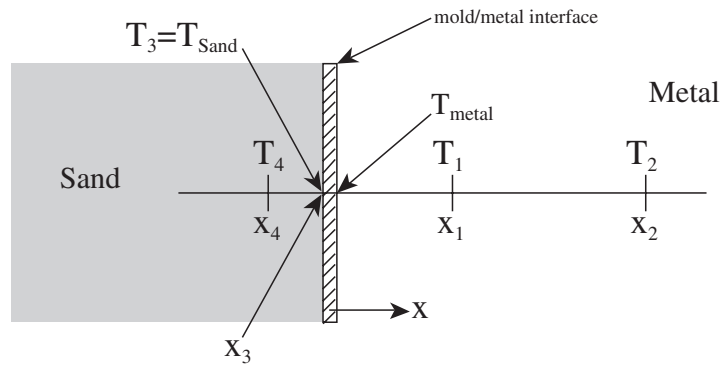

Fig. 2 Schematic diagram showing arrangement of temperature-measurement points near mold/metal interface.

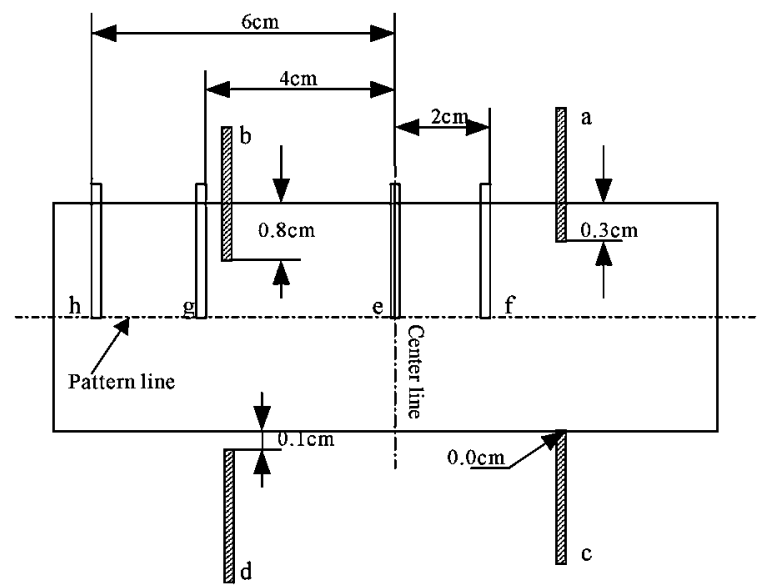

Fig. 3 Distribution of temperature measurement positions: (1) a, b, c and d denote measurement positions near the mold/metal interface, while (2) h, $\mathrm{g}$, $\mathrm{i}$, e and $\mathrm{f}$ denote measurement positions along the pattern line of the casting.

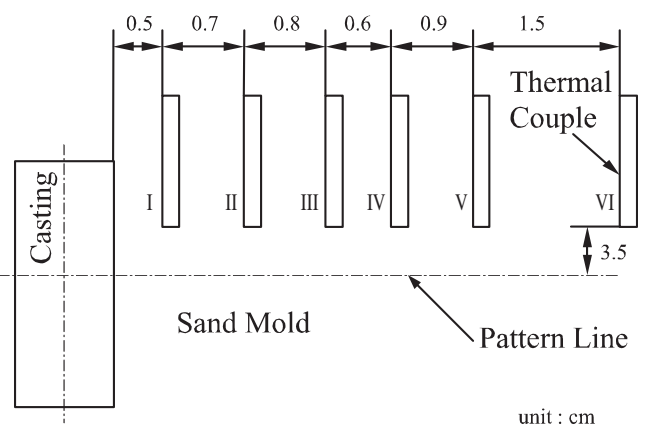

Fig. 4 Distribution of measurement points used to evaluate temperature within green-sand mold.

Fig. 2) can be measured directly using a thermocouple (i.e. point $\mathrm{c}$ in Fig. 3). As shown in Fig. 3, the temperature within the interior of the molten casting was measured using five thermocouples spaced along the pattern line of the casting (denoted by positions, h, g, i, e and f, respectively.) Finally, the temperature distribution within the green-sand mold in the radial direction was measured using a total of six nonuniformly spaced thermocouples, as shown in Fig. 4.

\subsection{Effective heat transfer coefficient, $h$}

In practical green-mold casting applications, a non-perfect contact exists at the mold/metal interface as a result of the 
surface roughness of the mold, differences in the thermal expansion coefficients of the metal and the mold, respectively, the vaporization of impurities in the mold, and so forth. Accordingly, the heat flux transferred through the mold/metal interface must be calculated using an effective heat transfer coefficient. The analysis performed in this study considers four different definitions of the effective heat transfer coefficient, namely

(1) The heat flux is discontinuous at the mold/metal interface as a result of the imperfect contact between the two surfaces. The heat fluxes from the two sides of the interface are balanced by the effective heat transfer coefficient, $h_{1}$, written as

$$
h_{1}=\left(q_{\text {metal }}-q_{\text {sand }}\right) /\left(T_{\text {metal }}-T_{\text {sand }}\right) .
$$

Note that since this definition is somewhat controversial and inconvenient to apply, it is mentioned here only for reference purposes.

(2) Most studies presented in the literature assume the presence of quasi-steady state conditions at the interface, ${ }^{8,11)}$ i.e. the heat flux flowing out of the metal is equal to that which flows into the mold. Under these conditions, the effective heat transfer coefficient, $h_{2}$, is given by

$$
h_{2}=q_{\text {metal }} /\left(T_{\text {metal }}-T_{\text {sand }}\right) .
$$

(3) The heat transfer process is confined primarily to the immediate vicinity of the mold/metal interface, and thus the temperature varies only slightly in regions of the sand mold far from the casting. This is easily verified via direct experimental measurements of the temperature variation in the sand mold, and is evidenced by the layer-like structure of the sand mold following the casting process, i.e. a burned black region adjacent to the casting, followed by a dry region, a moist region, and finally a non-affected zone, respectively. In addition, since sand has a low conductivity, the solidification process is analogous to the cooling of a high-temperature body in air. Therefore, the effective heat transfer coefficient, $h_{3}$, can be obtained by applying Newton's law to the boundary condition of the casting, i.e.

$$
h_{3}=q_{\text {metal }} /\left(T_{\text {metal }}-T_{\text {air }}\right),
$$

where $T_{\text {air }}$ is the temperature of the sand mold at a position far from the casting, i.e. room temperature.

(4) The heat transferred into the green-sand mold by the molten casting causes the moisture content within the mold to vaporize. As a result, a discernible interface is formed between the dry sand and the green sand, which gradually extends away from the mold/metal interface during the solidification process. In this case, the effective heat transfer coefficient, $h_{4}$, is derived by using the temperature of the dry/green sand interface $\left(T_{\mathrm{v}}\right)$ in place of the room temperature $\left(T_{\text {air }}\right)$ in eq. (3), i.e.

$$
h_{4}=q_{\text {metal }} /\left(T_{\text {metal }}-T_{\mathrm{v}}\right) .
$$

Note that $T_{\mathrm{v}}$ is generally taken as the vaporization temperature of water $\left(=100^{\circ} \mathrm{C}\right)$.

\subsection{Numerical analysis of metal and sand mold temper- ature distributions}

In the numerical analysis procedure, the surface temperature of the casting, i.e. $T_{\text {metal }}$, was determined by applying an inverse method to the experimental temperature measurements obtained in the interior of the casting. In the solution procedure, the temperature $T_{1 \text {,cal }}$ was calculated using an initial estimated value of $T_{\text {metal }}$ and the boundary condition $T_{2}$ obtained experimentally (see Fig. 2). The calculated value, $T_{1 \text {,cal }}$, was then compared to the experimental measurement, $T_{1 \text {,exp }}$. If the relative difference of $T_{1, \text { cal }}$ and $T_{1, \exp }$ was greater than 0.001, a new estimated value of $T_{\text {metal }}$ was supplied, and a new calculated temperature, $T_{1, \text { cal }}$, was determined. The iteration process continued until the relative difference was less than 0.001 . The inversely computed result of $T_{\text {metal }}$ was compared with that extrapolated from the measured data of $T_{1}$ and $T_{2}$.

In the sand mold numerical analysis procedure, the temperature of the sand mold at the mold/metal interface ( $T_{\text {sand }}$ shown in Fig. 2) should be determined in advance of calculating the temperature distribution of the green-sand mold. In this work, the temperature data of $T_{\text {sand }}$ are taken from the experimentally measured ones. Afterwards, the position of the dry/green interface can be determined as well as the temperature of the sand mold. The displacement of the dry/wet interface in the green-sand mold is given by

$$
-k \frac{\partial T}{\partial \mathrm{x}}=L \rho W \frac{\partial \zeta}{\partial \mathrm{t}},
$$

where $\zeta(\mathrm{t})$ is the position of the dry/wet interface with respect to the temperature $T_{\mathrm{v}}, k$ and $\rho$ are the thermal conductivity and density of the dry sand, respectively, and $L$ is the latent heat of water. Finally, $W$ is the moisture content in percentage terms at the vaporization interface. According to Marek, ${ }^{7)} W$ is given by $W=\left[W_{0} C p_{\text {water }}+(100-\right.$ $\left.\left.W_{0}\right) C p_{\text {dry }}\right] \frac{T_{\mathrm{v}}-T_{\mathrm{i}}}{L}$, where $W_{0}$ is the initial moisture content in the sand mold, $T_{\mathrm{i}}$ is the initial temperature of the sand mold, $C p_{\text {water }}$ is the specific heat of water, $C p_{\text {dry }}$ is the specific heat of dry sand, and $T_{\mathrm{v}}\left(=100^{\circ} \mathrm{C}\right)$ is the vaporization temperature of water. The numerical method used in this section is the finite difference method. The effective specific method is utilized to handle the latent heat of phase change.

\subsection{Finite element analysis of heat transfer}

The temperature contours within the cast cylindrical aluminum billet were simulated over time using FIDAP finite element software. FIDAP is unsuitable for modeling systems with complicated boundary conditions such as those associated with the models relating to effective heat transfer coefficients $h_{1}$ and $h_{2}$. Thus, the temperature distribution within the casting was simulated using effective heat transfer coefficient $h_{3}$. In the calculation, the effective specific method is also used to deal with the release of latent heat during the solidification process.

\section{Results and Discussions}

\subsection{Experimental cooling curves in metal casting}

Figure 5 presents the cooling curves within the aluminum casting as measured by the thermocouples positioned along 


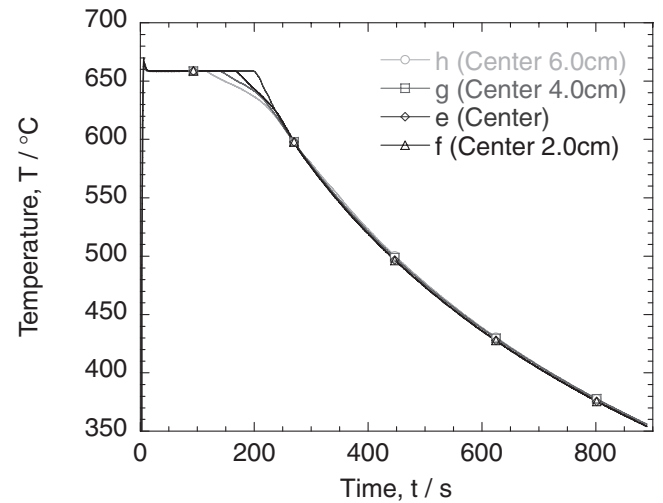

(a) 1000 seconds after pouring

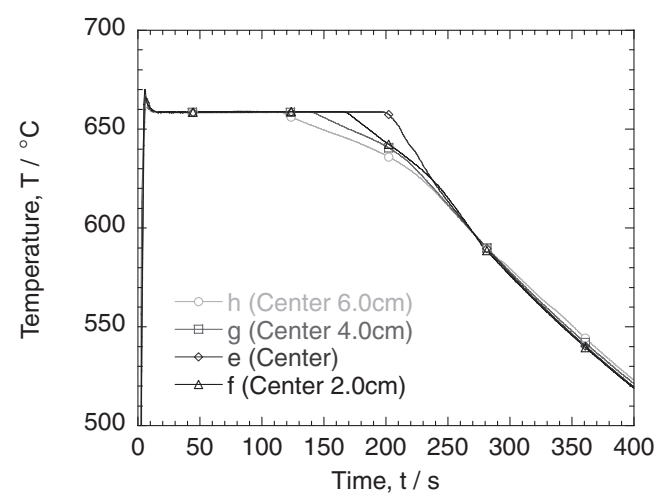

(b) 400 seconds after pouring

Fig. 5 Experimental cooling curves along centerline of aluminum casting, (a) 1000 seconds after pouring, (b) 400 seconds after pouring.

the centerline shown in Fig. 3. The results show that following the pouring process, the temperature of the casting drops virtually immediately to $660^{\circ} \mathrm{C}$, i.e. the melting point (freezing point) of aluminum. As the metal solidifies it releases latent heat, and thus the temperature remains approximately constant. From inspection, it can be seen that the time required for the aluminum casting to fully release the latent heat is around $t \cong 200$ seconds. During this period, the metal near the mold/metal interface releases its latent heat more rapidly than that in the interior of the casting. Therefore, as shown in Fig. 5(b), the temperature measurements obtained at positions closer to the sand mold (e.g. $6 \mathrm{~cm}$ from the center) remain constant for a shorter period of time than those closer to the center of the casting. Once the latent heat has been completely released, it can be seen that the temperature decreases rapidly with increasing time. Finally, the aluminum casting attains a uniform temperature as a result of its high thermal conductivity.

\subsection{Analysis of effective heat transfer coefficients}

In this study, the effective heat transfer coefficient at the mold/metal interface was evaluated based on the interfacial metal temperature $T_{\text {metal }}$ (see eqs. (1)-(4)), where $T_{\text {metal }}$ was obtained from temperature measurements $T_{1}$ and $T_{2}$ using either an extrapolation method or a numerical inverse technique. As expected, Fig. 6 shows that $T_{\text {metal }}$ varies as a function of time. The temperature values estimated by these

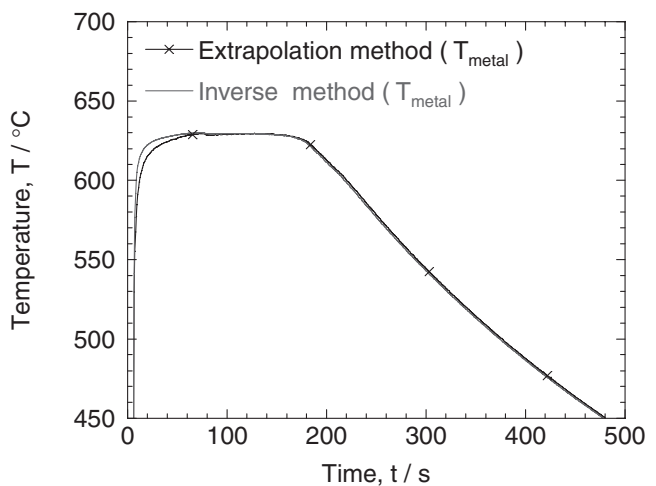

Fig. 6 Comparison of $T_{\text {metal }}$ as calculated by inverse numerical method and experimental extrapolation method.

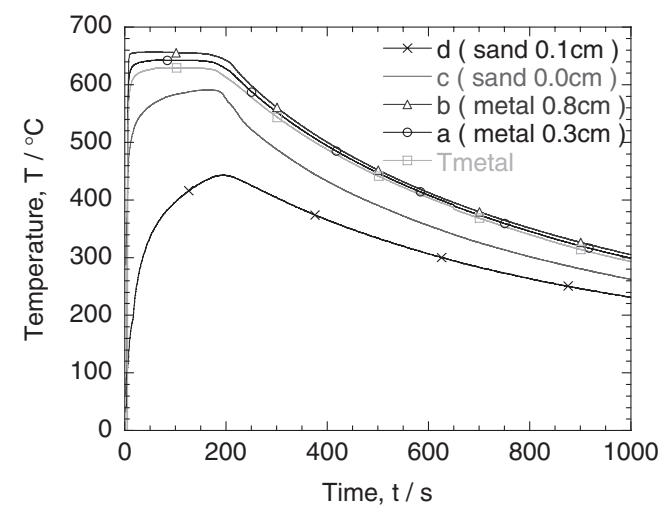

Fig. 7 Experimental cooling curves in interfacial region of casting system.

two methods are very close to each other. From inspection, the average discrepancy between the two sets of results is determined to be just $0.12 \%$. However, the inversely calculated temperature is higher than that of the extrapolation method, since the former one considers the effect of latent heat.

Figure 7 shows the experimental results obtained for the cooling curves in the region of the cylindrical casting near the mold/metal interface. It is observed that the cooling curve corresponding to a measurement point located $0.3 \mathrm{~cm}$ from the mold/metal interface is similar to the curves shown in Fig. 5 for the centerline temperature distributions. The temperature at this point lies below the freezing temperature on the temperature rise curve after pouring, which implies that the temperature near the interface falls rapidly following the pouring process. Furthermore, it can be seen that the temperature curves corresponding to measurement positions in the sand mold rise rapidly in the interfacial region and show no evidence of a stagnation phenomenon at the vaporization temperature (i.e. $100^{\circ} \mathrm{C}$ ).

Figure 8 clearly shows the effective heat transfer coefficient has a transient response below 400 seconds, but stabilizes thereafter. In every case, it can be seen that $h$ has its highest value immediately after the pouring process due to the perfect contact between the molten metal and the sand mold and the large temperature differential. Thereafter, the value of $h$ drops to a local minimum, before rising once again as a result of a vaporization of the moisture 


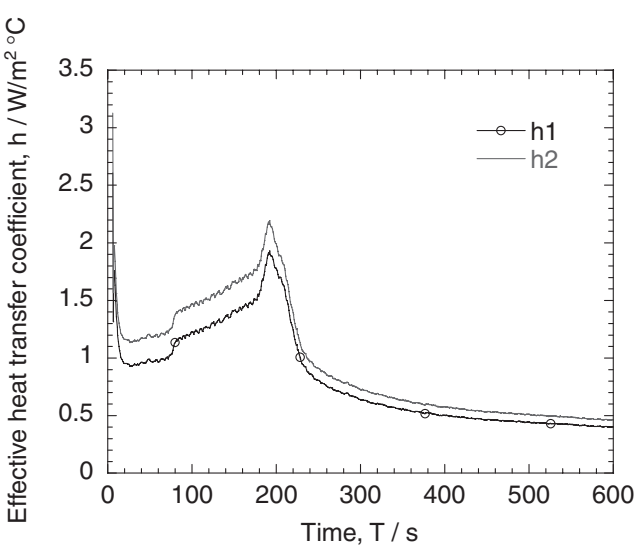

(a) Variation of $h_{1}$ and $h_{2}$ over time

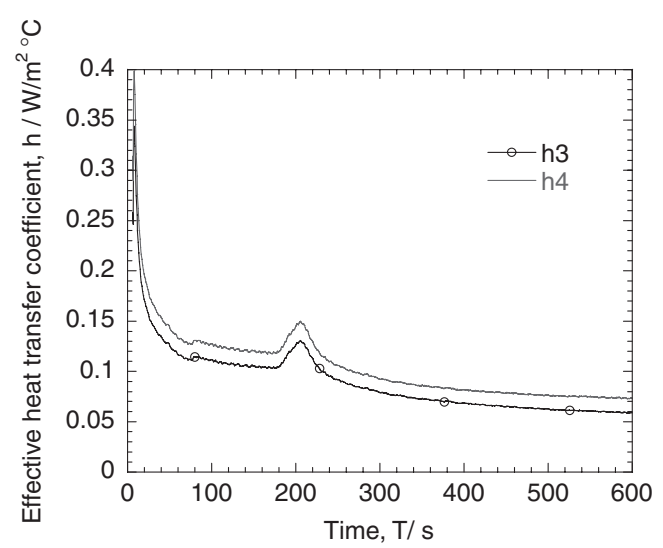

(b) Variations of $h_{3}$ and $h_{4}$ over time

Fig. 8 Comparison of effective heat transfer coefficients $h_{1}, h_{2}, h_{3}$ and $h_{4}$ over time, (a) Variation of $h_{1}$ and $h_{2}$ over time, (b) Variations of $h_{3}$ and $h_{4}$ over time.

content within the sand mold. The vapor has sufficient pressure to penetrate the sand mold and absorb more heat. Subsequently, the value of $h$ falls once again as a result primarily of the thermal resistance induced by the formation of an air gap at the mold/metal interface as the casting solidifies and contracts. Note that during the solidification process, the dry/wet interface moves progressively further away from the casting. This causes the vapor to condense outside the dry sand zone and therefore prompts a further reduction in the heat transfer (i.e. a further reduction in the value of $h$ ).

Since the value of the effective heat transfer coefficient depends strongly on local environmental factors, it is conveniently analyzed using a lumped-capacity model. Assuming the temperature within the metal casting to be uniformly distributed, the overall heat balance within the casting system over the course of the casting process can be expressed as

$$
h_{\mathrm{i}}=\frac{\left[C p_{\ell}\left(T_{\mathrm{p}}-T_{\mathrm{f}}\right)+L_{\mathrm{f}}+C p_{\mathrm{s}}\left(T_{\mathrm{f}}-T_{\text {final }}\right)\right]}{\int_{0}^{t_{\text {tinal }}} \mathrm{A}\left(T_{\mathrm{i}}-T_{\text {ref }}\right) \mathrm{dt}}
$$

where $h_{\mathrm{i}}$ is the effective heat-transfer coefficient and the subscript $i$ has the form
Table 1 Comparison of $h_{\mathrm{i}}\left(W / \mathrm{m}^{2 \circ} \mathrm{C}\right)$ for aluminum.

\begin{tabular}{|c|c|c|c|c|}
\hline$h_{5}$ & $h_{6}$ & \multicolumn{2}{|c|}{$\left(h_{5}+h_{6}\right) / 2$} & $\begin{array}{c}h_{3} \\
\text { average value }\end{array}$ \\
\hline 0.0795 & 0.0831 & \multicolumn{2}{|c|}{0.0813} & 0.0817 \\
\hline$h_{7}$ & $h_{8}$ & \multicolumn{2}{|c|}{$\left(h_{7}+h_{8}\right) / 2$} & $\begin{array}{c}h_{4} \\
\text { average value }\end{array}$ \\
\hline 0.0982 & 0.1026 & \multicolumn{2}{|c|}{0.1004} & 0.1004 \\
\hline & $\left\{\begin{array}{l}5 \\
6 \\
7 \\
8\end{array}\right.$ & $\begin{aligned} T_{\mathrm{i}} & =T_{\mathrm{c} \ell} \\
T_{\mathrm{i}} & =T_{\text {metal }} \\
T_{\mathrm{i}} & =T_{\mathrm{c} \ell} \\
T_{\mathrm{i}} & =T_{\text {metal }}\end{aligned}$ & $\begin{array}{l}T_{\text {ref }} \\
T_{\text {ref }} \\
T_{\text {ref }} \\
T_{\text {ref }}\end{array}$ & \\
\hline
\end{tabular}

where $T_{\mathrm{c} \ell}$ is the temperature of the casting at the center point, $T_{\mathrm{p}}$ is the pouring temperature, $T_{\mathrm{f}}$ is the melting temperature, $t_{\text {final }}$ is the calculated value of the final time, $T_{\text {final }}$ is the temperature at the final time, $\mathrm{A}$ is the surface area of the casting, and $\mathrm{V}$ is the volume of the casting.

The values of $h_{\mathrm{i}}$ are summarized in Table 1 . It is observed that the average value of $h_{3}$ is similar to $h_{5}$ and $h_{6}$, while the average value of $h_{4}$ is similar to $h_{7}$ and $h_{8}$. This finding confirms that the lumped-capacity method provides a feasible technique for estimating the effective heat transfer coefficient within the aluminum/green-sand mold casting system without the need for experimental temperature measurements in the interfacial region.

\subsection{Temperature distribution in green-sand mold}

Figure 9 illustrates the experimental heating curves obtained in the green-sand mold at various distances from the mold/metal interface. As shown, the temperature increases from room temperature to $T=100^{\circ} \mathrm{C}$ and then remains at this temperature as the vapor heat is released (see measure point I, II, III and IV). For example, the temperature at $0.5 \mathrm{~cm}$ (see measure point I) from the interface increases to $100^{\circ} \mathrm{C}$ then gradually rise to more than $350^{\circ} \mathrm{C}$. Once the vapor heat has been fully released, the results show that the local temperature rises continuously with an increasing elapsed time. Comparing the various temperature profiles shown in the figure, it is determined that the vapor heat release process takes shorter time in the regions of the mold closer to the mold/metal interface.

The corresponding computed heating curves at temperature-measured points in the sand mold are shown in Fig. 10. The relative errors between the measured and the calculated temperatures at the measurement positions in the green-sand mold are summarized in Table 2. From Fig. 9, 10 and Table 2 , it could be found that the computed heating curves are similar to the experimental ones.

Figure 11 presents experimentally measured temperature distributions along the measured points in the green-sand mold, which are indicated in Fig. 4. From the figure, it can be seen that the temperature in the green-sand mold increases rapidly from room temperature following the pouring process and then decreases gradually with an increasing elapsed time. It is also observed that the temper- 


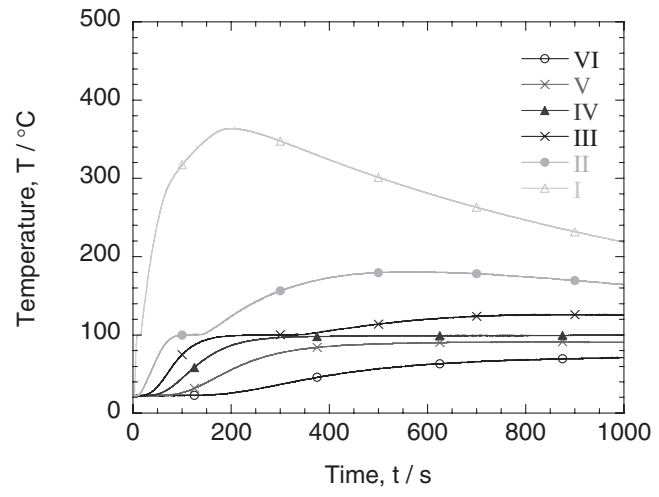

Fig. 9 Experimentally measured heating curves at those temperaturemeasured points in the green-sand mold indicated in Fig. 4.

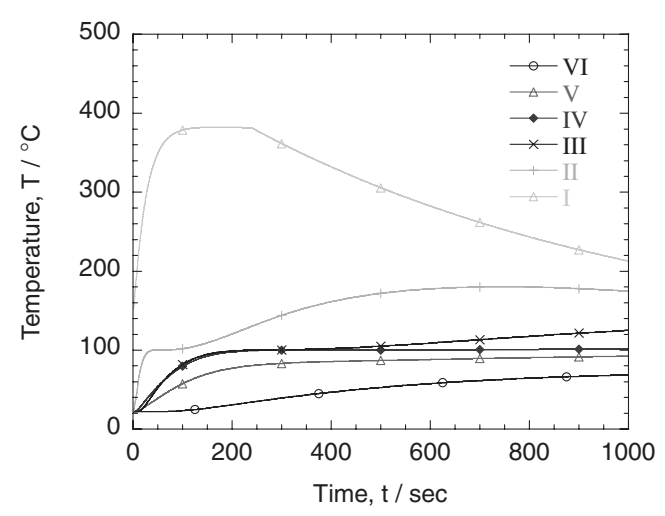

Fig. 10 Numerically computed heating curves at those temperaturemeasured points in the green-sand mold indicated in Fig. 4.

Table 2 Relative errora between the measured and the calculated temperatures at the measured points in the green-sand mold.

\begin{tabular}{c|cccccc}
\hline Mold site & $0.5 \mathrm{~cm}$ & $1.2 \mathrm{~cm}$ & $2.0 \mathrm{~cm}$ & $2.6 \mathrm{~cm}$ & $3.5 \mathrm{~cm}$ & $5.0 \mathrm{~cm}$ \\
Error value & I & II & III & IV & V & VI \\
\hline$\varepsilon$ & $0.74 \%$ & $0.16 \%$ & $0.22 \%$ & $0.26 \%$ & $0.31 \%$ & $0.13 \%$ \\
\hline
\end{tabular}

ature rise at the measurement points closer to the mold/ metal interface is particularly pronounced due to the relatively greater amount of heat flux input to the sandmold in the interfacial region.

\subsection{FIDAP simulation results}

Using the effective heat transfer coefficient $h_{3}$, a FIDAP simulation was performed to calculate the temperature field within the cylindrical aluminum casting during the solidification process. The computation results are shown in Fig. 12, in which the temperature distributions of the metal varying with time are illustrated. From the simulation results, it can be found that complete solidification occurs 220 seconds after the completion of the pouring process. This result is in reasonable agreement with the solidification time of 200 seconds observed in the physical casting experiment. Moreover, the simulation results indicate that the temperature within the casting reduces rapidly once complete solidification has occurred.

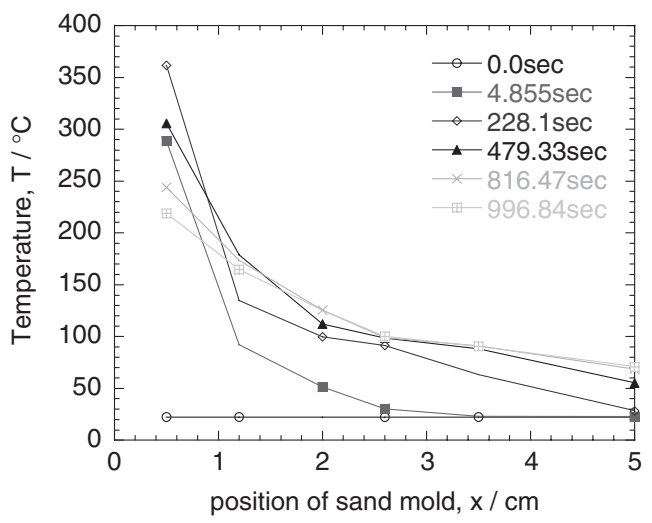

Fig. 11 Experimentally measured temperature distributions along the measured points in the green-sand mold indicated in Fig. 4.

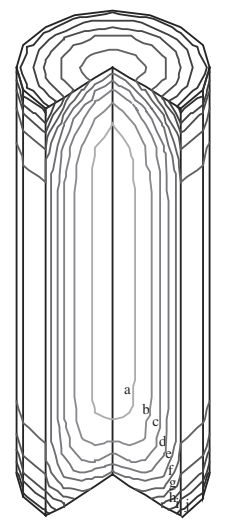

(a) time $=100$ seconds

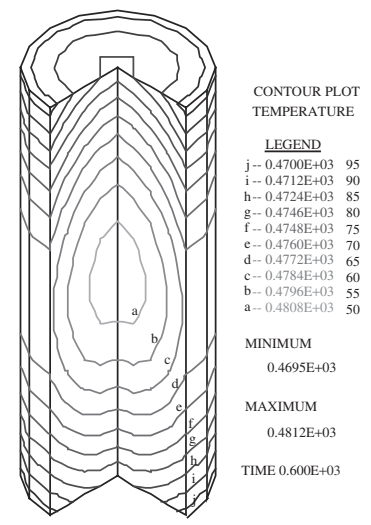

(c) time $=500$ seconds

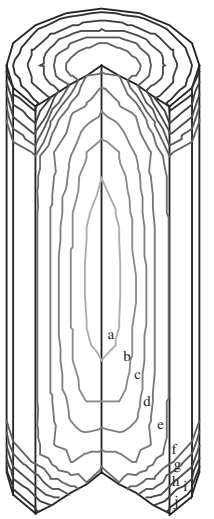

CONTOUR PLOT CONTOUR PLOT

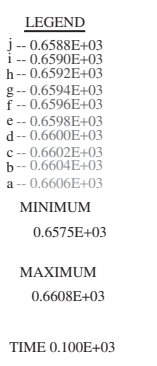

(b) time $=200$ seconds

TEMPERATURE

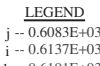

$-0.6191 \mathrm{E}+03$ $\mathrm{g}-0.06245 \mathrm{E}+03$
$\mathrm{f}--0.6299 \mathrm{E}+0$ e $-0.6353 \mathrm{E}+0.3$

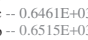
MINIMUM $0.6081 \mathrm{E}+03$ MAXIMUM $0.6590 \mathrm{E}+03$ TIME $0.300 \mathrm{E}+03$

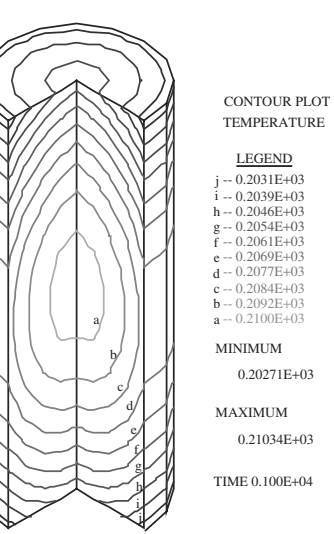

Fig. 12 FIDAP-simulated temperature distributions of the metal varying with time, (a) time $=100$ seconds, (b) time $=200$ seconds, (c) time $=500$ seconds, $(\mathrm{d})$ time $=1000$ seconds.

\section{Conclusions}

This study has performed an experimental and numerical investigation into the effective heat transfer coefficient at the interface between a cylindrical aluminum casting and a green-sand mold. In the temperature measurement, a symmetric arrangement of thermocouples is proposed and proven to be feasible, which can reduce the influence of heat-transfer and solidification characteristics in a casting 
experiment caused by the close-spaced thermocouples. The cooling curves have shown that the temperature of the molten metal falls rapidly to the melting point (freezing point) following the pouring process, and then stays at this temperature as the latent heat is released. It has been shown that the rate of latent heat release increases with a decreasing distance from the mold/metal interface. In addition, the cooling curves have shown that the aluminum casting cools to a uniform temperature as a result of its high thermal conductivity.

The effective heat transfer coefficient $(h)$ at the mold/ metal interface has been evaluated based upon the temperature of the molten metal at the mold/metal interface $\left(T_{\text {metal }}\right)$ as calculated using an extrapolation technique and an inverse scheme. The results have shown that $h$ attains its highest value immediately after the pouring process due to the perfect contact formed between the molten metal and the sand mold and the high temperature differential. Thereafter, $h$ drops to a local minimum value before rising once again as a result of moisture vaporization. Finally, the value of $h$ decreases progressively as the result of the thermal resistance induced by the formation of an air gap at the mold/metal interface. It has been found that the values of $h_{3}$ and $h_{4}$ do not vary greatly over time, and thus the lump capacity method provides a convenient means of estimating the effective heat transfer coefficient.

A good agreement has been found between the numerical results and the experimental results for the temperature distributions within the green-sand mold. Both sets of results have shown that the temperature of the sand mold rises from room temperature to the moisture vapor point and then stays at this temperature as the vapor heat is released. The results have also shown that the vapor heat release process takes shorter time in the regions of the mold closer to the mold/ metal interface.

Finally, the effective heat transfer coefficient $h_{3}$ has been used to perform a FIDAP simulation of the temperature field within the cylindrical aluminum casting as a function of time during the solidification process. The predicted value of the solidification time ( 220 seconds) was found to be in good agreement with that observed experimentally (200 seconds).

\section{REFERENCES}

1) S. Ho and R. D. Pehlke: Metall. Trans. B 16 (1985) 585-594.

2) X. C. Zeng and R. D. Pehlke: AFS Trans. 93 (1985) 275-282.

3) T. X. Hou and R. D. Pehlke: AFS Trans. 94 (1986) 129-136.

4) K. Kubo and R. D. Pehlke: Metall. Trans. B 17 (1986) 903-911.

5) H. L. Tsai, K. C. Chiang and T. S. Chen: AFS Trans. 96 (1988) 191196.

6) T. S. Shih, S. S. Hsiau and C. H. Hong: AFS Trans. 104 (1996) 481489.

7) C. T. Marek: AFS Trans. 71 (1963) 185-192.

8) P. A. Kobryn and S. L. Semiatin: Metall. Mater. Trans. B 32 (2001) 685-695.

9) Y. Nishida, W. Droste and S. Engler: Metall. Trans. B 17 (1986) 833844.

10) J. C. Hwang, H. T. Chuang, S. H. Jong and W. S. Hwang: AFS Trans. 144 (1994) 877-883.

11) D. J. Browne and D. O'Mahoney: Metall. Mater. Trans. A 32 (2001) 3055-3063.

12) F. Chiesa: AFS Trans. 98 (1990) 193-200. 